\title{
Magnetic resonance imaging of abnormal ventricular septal motion in heart diseases: a pictorial review
}

\author{
Cristina Méndez • Rafaela Soler • Esther Rodriguez • \\ Marisol López • Lucia Álvarez • Noela Fernández • \\ Lorenzo Montserrat
}

Received: 25 September 2010 /Revised: 4 January 2011 /Accepted: 4 April 2011 /Published online: 17 April 2011

(C) European Society of Radiology 2011

\begin{abstract}
The purpose of this article is to illustrate the usefulness of MR imaging in the clinical evaluation of congenital and acquired cardiac diseases characterised by ventricular septal wall motion abnormality. Recognition of the features of abnormal ventricular septal motion in MR images is important to evaluate the haemodynamic status in patients with congenital and acquired heart diseases in routine clinical practice.
\end{abstract}

Electronic supplementary material The online version of this article (doi:10.1007/s13244-011-0093-4) contains supplementary material, which is available to authorized users.

C. Méndez $\cdot$ R. Soler $\cdot$ E. Rodriguez $(\bowtie) \cdot$ M. López $\cdot$

L. Álvarez $\cdot$ N. Fernández

Department of Radiology,

Complejo Hospitalario Universitario A Coruña (CHUAC),

Xubias de Arriba 84,

15006 A Coruña, Spain

e-mail: esther.rodriguez@mundo-r.com

C. Méndez

e-mail: cristinamendezdiaz@hotmail.com

R. Soler

e-mail: rafaelasoler@telefonica.net

M. López

e-mail: marisol.lopez.rodriguez@sergas.es

L. Álvarez

e-mail: lucia.alvarez.devesa@sergas.es

N. Fernández

e-mail: noela.fernandez.guillan@sergas.es

L. Montserrat

Department of Cardiology,

Complejo Hospitalario Universitario A Coruña (CHUAC),

Xubias de Arriba 84,

A Coruña 15006, Spain

e-mail: lorenzo.monserrat.iglesias@sergas.es
Keywords Heart diseases $\cdot$ Heart function · Ventricular septum $\cdot$ Magnetic resonance imaging

\section{Introduction}

The interventricular septum (IVS) is an important structure that not only plays a direct role in bi-ventricular function, but can also reflect changes in the function of either ventricle, exhibiting abnormal configurations and motions that have physiological and diagnostic value [1].

Many conditions can cause abnormal motion of the ventricular septum. Although characteristics have been described to help differentiate these entities, its appearance on echocardiography may be similar [2]. Cardiac MRI can evaluate the motion of cardiac structures, accurately assess the right and left ventricular function, and identify myocardial viability in a single examination.

This article reproduces MR images of a wide range of congenital and acquired heart diseases characterised by abnormal configuration and motion of the ventricular septum.

\section{Position and geometry of the ventricular septum}

Under normal conditions, the septum has a right convexity, and this configuration is maintained during the cardiac cycle. Because the two ventricles compete for space within the pericardium and share common myocardial fibres in the septal region, filling in one ventricle affects filling in the other, because of septal shift. This is a normal physiological phenomenon known as ventricular interdependence, which is altered by the pressure changes during ventricle filling [ 1 , 3]. Inspiration enhances early right ventricular (RV) filling while the opposite occurs during expiration, which leads to 
a small septal excursion in normal subjects, and in a minority to inspiratory septal flattening dependent upon the depth of respiration [3].

Paradoxical septal motion (PSM) is the abnormal movement of the IVS towards the left ventricle despite normal thickening. The abnormal ventricular septal motion can occur with right ventricle to left ventricle pressure gradient change, when the pressure overcomes the myocardial stress and contractile force or there is a loss of myocardial contractility $[1,4]$.

Paradoxical septal motion is a common finding after uncomplicated cardiac surgery, and has been recognised in patients with right-sided regurgitant lesions, left-to-right shunts, pulmonary hypertension, mitral stenosis, constrictive pericarditis and heart diseases with abnormal conduction. Several causes of PSM have been proposed, yet the exact mechanism remains unclear [5].

The ventricular septal motion can be visually assessed on short-axis and four-chamber cine-MR views in the two cardiac phases and, its position classified as normal, flattened or left bowing (convex towards the left ventricle; Fig. 1). A summary of the different types of abnormal septal motion in congenital and acquired heart diseases is presented in Table 1.

In the short-axis view, the abnormal septal motion can be easily quantified on the basis of the septal curvature. A negative value of septal curvature represents leftwards bowing of the septum into the left ventricular (LV) cavity, and a positive value indicates rightwards bowing or a more physiological septal contour $[3,6]$. In conjunction with the ratio of septal curvature, the relationship between the left ventricle and the right ventricle can be quantitated on the basis of the ratio between the LV antero-posterior dimension and the septo-lateral dimension at end systole or end diastole. This "eccentricity index" is abnormal when this ratio is more or less than 1.0 (Fig. 2) [7].

\section{Right ventricular volume overload}

Whereas in the short-axis view, the LV cavity maintains a circular profile throughout the cardiac cycle in normal subjects, in RV volume overload, the left ventricle assumes a progressively more D-shaped cavity as the ventricular septum flattens and progressively loses its convexity with respect to the centre of the RV cavity during diastole, with relative sparing of LV deformation at end-systole (Movie 1) [8].

The most common lesions associated with RV volume overload are left to right shunts, tricuspid regurgitation in the setting of Ebstein anomaly and pulmonary regurgitation in the setting of tetralogy of Fallot.

\section{Left to right shunts}

Atrial septal defects and partially anomalous pulmonary venous returns to systemic veins or directly to the right atrium result in left-to-right shunting, which when significant, leads to right atrial and ventricular enlargement and pulmonary artery dilatation. In these patients, the degree of flattening and leftwards septal bowing during diastole (Fig. 3) was related to the degree of volume overload [9].

Surgical closure should be considered in the short term for patients with a ratio of pulmonary-to-systemic blood flow $(\mathrm{Qp} / \mathrm{Qs})$ higher than 1.5, irrespective of age. After surgical closure, the persistence of right ventricular dilatation and paradoxical septal motion are common, with older age at surgery, systolic pulmonary artery pressure $>40 \mathrm{mmHg}$ and a ratio of pulmonary/systemic blood flow $>3$ [10].

\section{Pulmonary and tricuspid regurgitation}

Ebstein's anomaly is a common primary cause of tricuspid regurgitation characterised by varying degrees of dysplasia
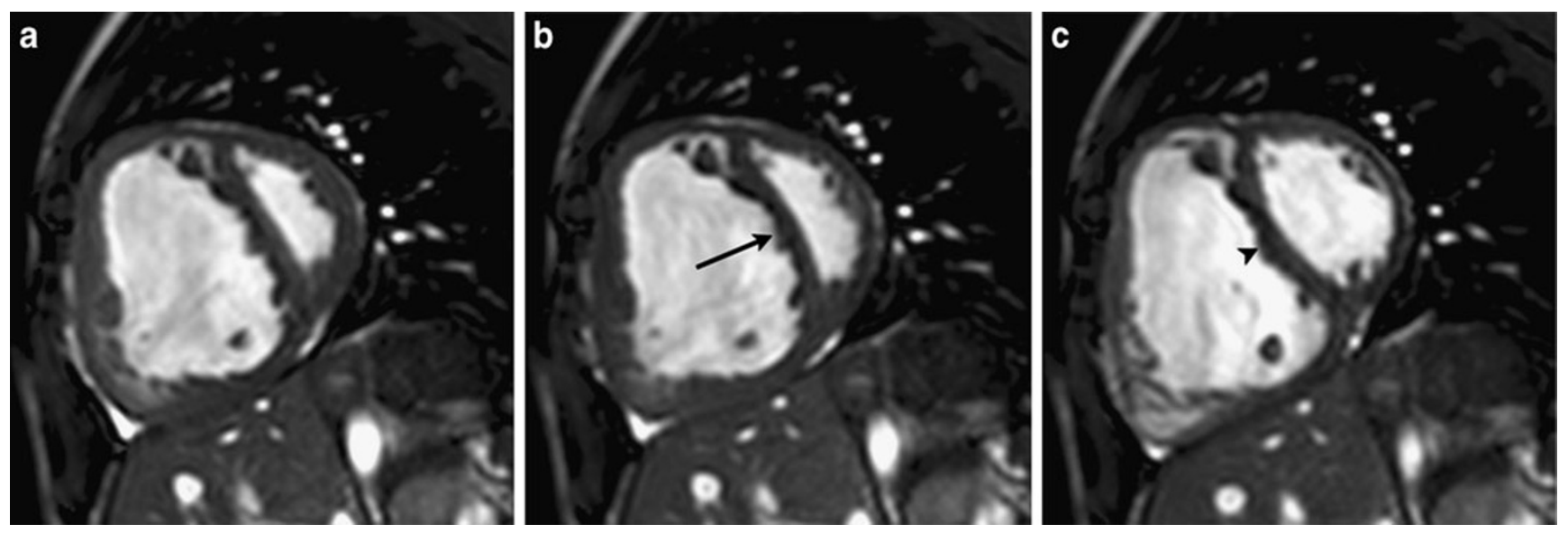

Fig. 1 A 61-year-old man with pulmonary hypertension and dyspnea at exertion. End systolic (a), and early (b) and end (c) diastolic shortaxis steady-state free precession cine MR images. During early filling, the septum (arrow) bows into the left ventricle and regains a normal appearance (arrowhead) at the end of diastolic filling. The septum is flattened at end systole 
Table 1 Types of paradoxical septal motion. RV=right ventricular; RVOT=right ventricular outflow tract; D-TGA= dextroposed transposition of the great arteries; ccTGA $=$ congenitally corrected transposition of the great arteries
Paradoxical septal motion

\begin{tabular}{|c|c|c|}
\hline Systolic & Systolic and diastolic & Diastolic \\
\hline Left bundle branch block & $\begin{array}{l}\text { RV volume overload (left to right shunts, } \\
\text { tricuspid and pulmonary regurgitation) }\end{array}$ & Pericardial constriction \\
\hline $\begin{array}{l}\text { Antero-septal infarction } \\
\text { Post-open heart surgery }\end{array}$ & $\begin{array}{l}\text { RV pressure overload (RVOT obstruction, } \\
\text { pulmonary hypertension, D-TGA after } \\
\text { atrial switch procedures and ccTGA end systole) }\end{array}$ & $\begin{array}{l}\text { Arrhythmogenic RV } \\
\text { cardiomyopathy }\end{array}$ \\
\hline
\end{tabular}

and displacement of the tricuspid valve leaflets into the RV [11]. The major haemodynamic consequence of tricuspid regurgitation is an increase in end-diastolic RV volume that leads to paradoxical septal motion during diastole. Although this abnormal septal motion is not specific to valve regurgitation, its presence is considered to be a distinct indirect echocardiographic and Doppler parameter for severe tricuspid regurgitation [12]. In some cases, right ventricular dilatation is so marked that the ventricular septum bulges leftwards during diastole, compressing the left ventricular chamber (Fig. 4). Episodic left ventricular outflow tract obstruction may be observed in extreme cases [11].

Tetralogy of Fallot (TOF) is the most common form of cyanotic congenital heart disease after the first year of life. Most patients with TOF nowadays undergo total repair early in life via closure of the ventricular septal defect and relief of RV outflow tract (RVOT) obstruction with good results.

Long-term follow-up studies have reported that following TOF repair, these patients have residual haemodynamic abnormalities, such as pulmonary regurgitation after RVOT patch augmentation, which leads to progressive RV dilatation, tricuspid regurgitation and secondary RV volume overload [7, 13]. This abnormal RV volume overload is associated with flattening (Movie 1) or leftwards septal bowing during diastole that causes alterations in left ventricular geometry and function (Fig. 5 and movie 1) [7].

Left ventricular dysfunction in patients following TOF repair was found to be the strongest predictor of poor clinical status. Proposed mechanisms include akinesia resulting from the ventricular septal defect patch, septal fibrosis, chronic volume loading from early palliative shunt creation, progressive mechanical interaction between an enlarged failing RV and the LV that is mediated through paradoxical septal motion, and myocardial injury at the time of repair [7, 13]. Right ventricular restoration in patients with severe right ventricular dilatation and underlying aneurysm or akinesia of the right ventricular outflow tract has proven to be an effective procedure to return the bowed non-functional septum to a central position that improves right-sided function [13].

This paradoxical septal motion may also be observed in asymptomatic postoperative patients following TOF repair without significant right ventricular volume. Prolongation of the QRS duration secondary to right bundle branch block has
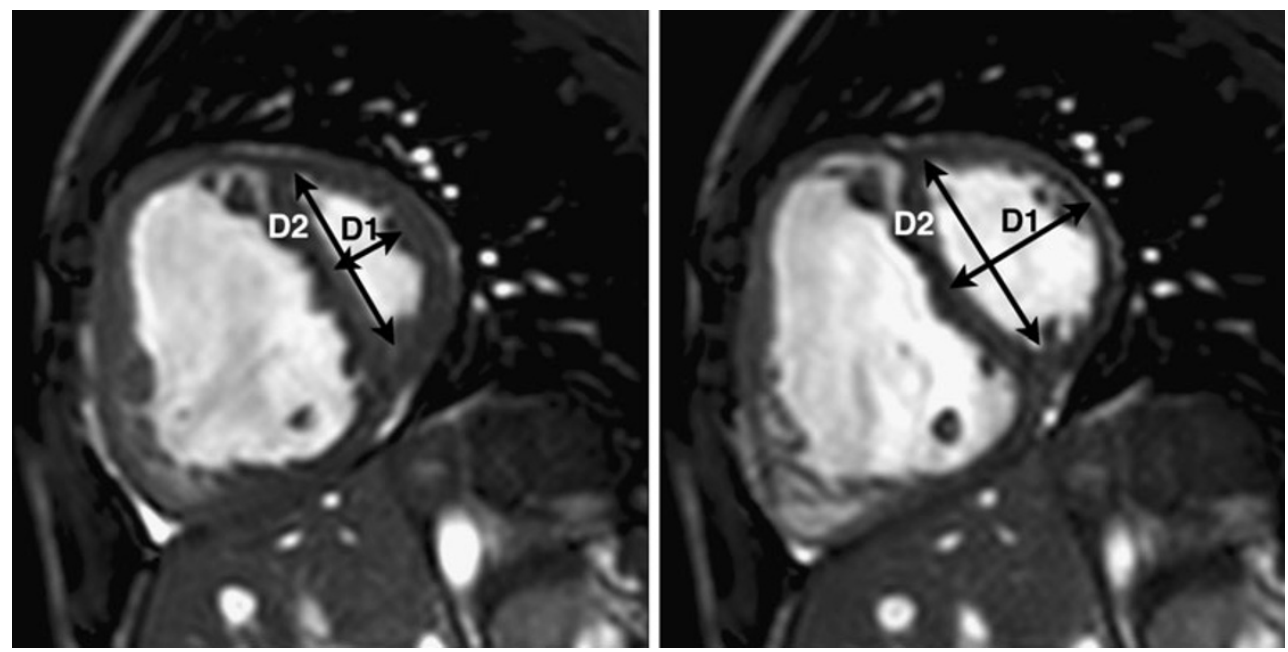

Fig. 2 Eccentricity index. Short-axis steady-state free precession cine MR images at end systole (left) and end diastole (right). The length of the short-axis diameter (perpendicular to the interventricular septum) from the left ventricular septal endocardium to the endocardium of the postero-lateral free wall is defined as D1. The length of the orthogonal short-axis diameter between the endocardial surfaces of the anterior and inferior LV free wall is defined as D2. Left ventricular eccentricity at end systole or end diastole was defined as the ratio of D2/D1. The eccentricity index was determined by dividing the D2/D1 throughout the cardiac cycle 
Fig. 3 A 38-year-old woman with symptoms of right heart failure. a Sagittal steady-state free precession cine MR image revealing direct communication (arrowhead) between the superior vena cava (asterisk) and the left atrium (LA), consistent with a sinus venous defect and an enlarged pulmonary trunk (arrow). The ratio between pulmonary and systemic flow $(\mathrm{Qp} / \mathrm{Qs})$ was 2.1 (not shown). b and $\mathbf{c}$ Coronal and oblique coronal maximum-intensity-projection images showing communication between the superior vena cava (asterisk) and the left atrium (arrowhead) and abnormal drainage of the right upper lobe pulmonary veins (arrow) to the superior vena cava (asterisk). d Short-axis steady-state free precession cine MR image obtained at systole revealing an enlarged right ventricle and leftward bowing of the ventricular septum (arrows)
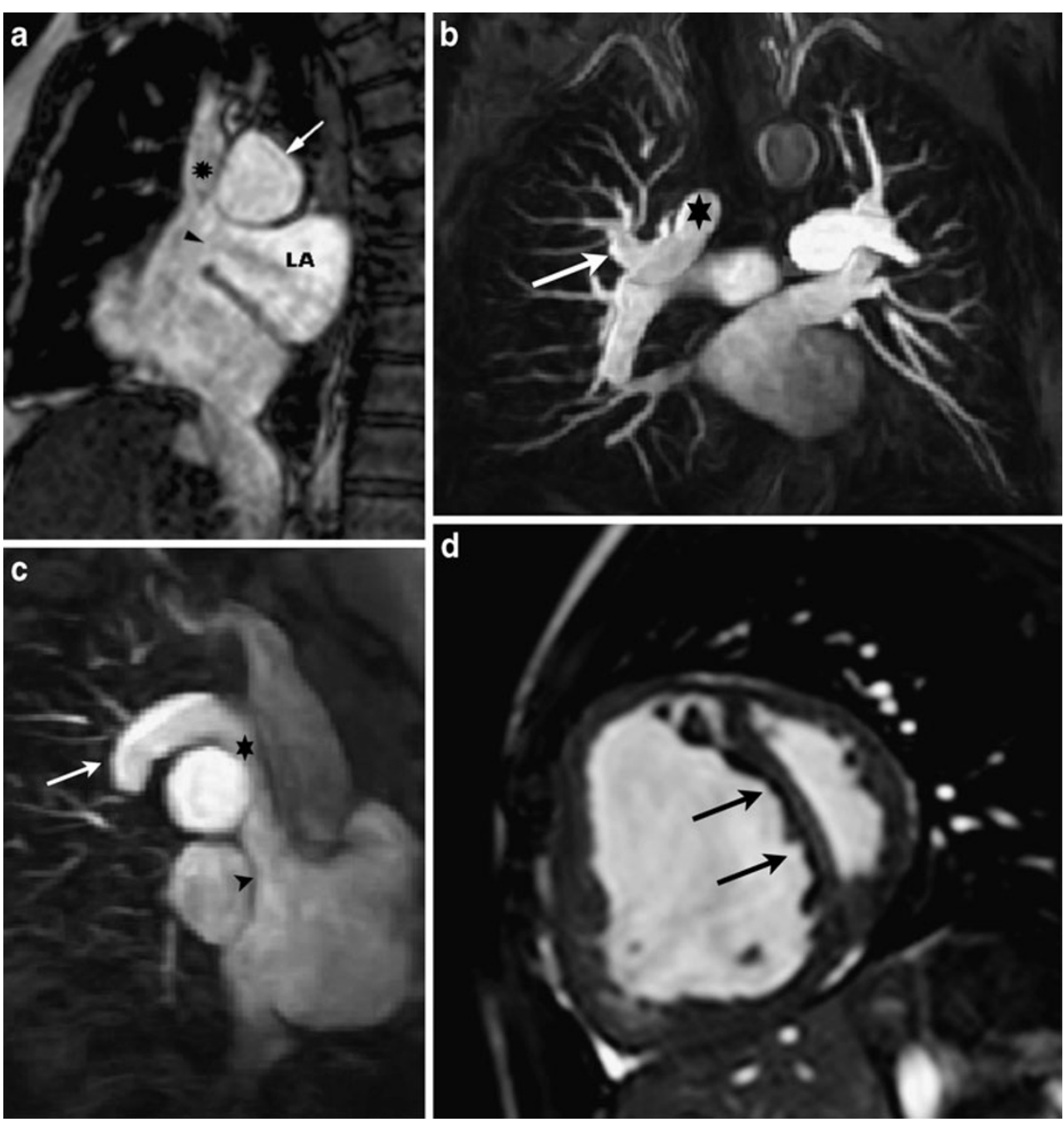

been related to the degree of reduced regional septal systolic function. Reduced LV systolic function in this group of patients is mainly secondary to diminished regional septal systolic function and the paradoxical septal motion [7].

\section{Right ventricular pressure overload}

Right ventricular pressure overload also distorts the normal circular short-axis geometry of the left ventricle by shifting the septum leftwards away from the centre of the right ventricle and towards the centre of the left ventricle, resulting in flattening or leftwards bowing of the ventricular septum predominantly during end systole and early diastole (Movie 2). The distortion in LV cavity at end systole due to septal flattening or leftwards bowing contributes to preserved systolic ventricular function [8].

The most common models of RV pressure overload are RV outflow tract (RVOT) obstruction and conditions with systemic RV pressures (pulmonary hypertension and systemic right ventricle).
Right ventricular outflow tract obstruction

Valvular pulmonary stenosis is found in patients with RVOT obstruction and is almost always congenital. Pulmonary stenosis, when significant, results in delayed ventricular filling and compensatory RV hypertrophy. RV systolic function is initially preserved with RV pressure overload, but diastolic dysfunction occurs as a consequence of myocardial hypertrophy and remodelling [14].

Cardiac MRI can show normal septal function or septal flattening or leftwards bowing throughout the cardiac cycle with most marked distortion of the left ventricle at early diastole. In addition, it is the gold standard method for quantifying RV size and function, and can provide additional information for assessing pulmonary stenosis and locating the exact area of obstruction [15].

After percutaneous pulmonary valve implantation, an increase in LV end-diastolic volume due to an improvement in early diastolic filling has been reported. This better LV filling correlates with more favourable septal motion [14]. 


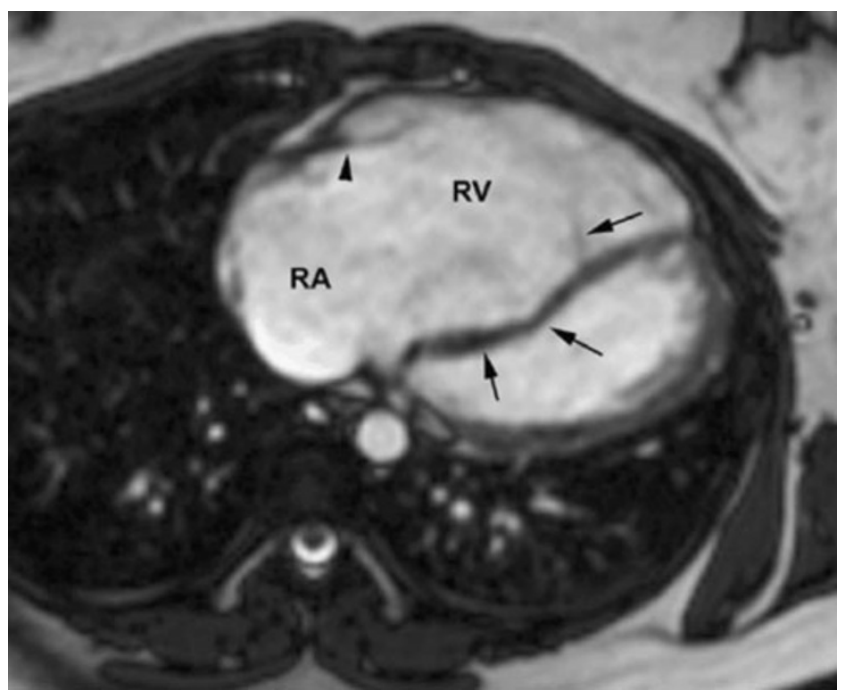

Fig. 4 A 24-year-old woman presenting with dyspnoea on exertion who had a history of exercise intolerance since childhood. Axial steady-state free precession cine MR images of the heart obtained at diastole showing an enlarged right atrium (RA) and right ventricle (RV), normal anatomical location tricuspid valve annulus and proximal portion of the anterior leaflet (arrowhead), inferiorly displaced septal leaflets (arrow) and leftward septal bowing of the atrialised right ventricle (arrows), which was accentuated during systole. The cause is an increase in right-sided blood volume and pressure. The tricuspid valve regurgitation rate calculated for this patient was $57 \%$ (not shown)

\section{Systemic right ventricular pressures}

\section{Pulmonary hypertension}

Pulmonary hypertension is characterised by a progressive increase in pulmonary vascular resistance from various congenital and acquired causes leading to right ventricular pressure overload and failure.

In patients with severe pulmonary hypertension, the RV pressure overload causes a decrease in the left-to-right transseptal pressure gradient. During early diastole the LV pressure drops to near zero to enable rapid LV filling, and the pressure in the RV prevails, pushing the septum away from the RV centre. This explains IVS flattening or inversion during the filling phase of diastole and the " $\mathrm{D}$ shape" of the left ventricle visible on short-axis views (Movie 2 and Fig. 6) [6].

The radius of septal curvature compared with the free wall curvature, measured with cardiac MR, has been recently proposed as a non-invasive index for estimating elevated RV systolic pressure and for explaining LV cavity deformation occurring in patients with pulmonary hypertension [15]. A leftwards septal bowing has only been observed at systolic pressures higher than $67 \mathrm{mmHg}$, and severe leftwards ventricular septal bowing is considered to be associated with a poor prognosis in pulmonary hypertension [6].
Left ventricular dysfunction in patients with pulmonary hypertension can be caused by synchronous coronary disease or a congenital defect, or can be secondary to the haemodynamic effects of pulmonary hypertension on the left ventricle. The increase in pulmonary vascular resistance causes a decrease in RV stroke volume and, consequently, the LV filling and LV stroke volume. In addition, the septal bowing further reduces the LV volume in early diastole, thus limiting the LV filling process during the most important phase of rapid filling [6]. Patients with pulmonary hypertension and LV impairment have a poor prognosis, tend to worsen and may require a double lungheart transplant.

\section{Systemic right ventricle}

A morphological right ventricle in the systemic position in adulthood is most commonly encountered in patients with congenitally corrected transposition of the great arteries (ccTGA) and those with dextro transposition of the great arteries (D-TGA) following atrial switch procedures (Mustard or Senning). The result is chronic RV pressure overload. Over time, both conditions may lead to RV dysfunction, and often this becomes a major clinical concern $[16,17]$.

In both of these conditions, the presence of significant tricuspid regurgitation and/or RV dysfunction is associated with a significantly higher mortality. The factors responsible for this remain unclear $[16,17]$.

In patients with ccTGA and D-TGA following the atrial switch procedure, in which the right ventricle is exposed to systemic pressure and the left ventricle is exposed to only pulmonary pressure, the ventricular septum can bow into the left ventricle during end-systole (Fig. 7). RV dysfunction and complete heart block are some of the causes implicated in the leftwards septal shift $[16,17]$.

This abnormal septal configuration could also contribute to tricuspid regurgitation in both conditions as the ventricular septum bows into the left ventricle and the septal attachments of the tricuspid valve are pulled away from its annulus. These geometric alterations potentially lead to a more inferior zone of coaptation, less overlap of the tricuspid valve leaflets and progressive tricuspid regurgitation [16].

It has been observed that tricuspid regurgitation increases after conventional or physiological repair, in which the tricuspid valve is left in the systemic circulation, and tricuspid regurgitation decreases after procedures that increase LV pressure, such as pulmonary artery banding. The clinical significance of these findings suggests that pulmonary artery banding may be effective as an isolated treatment for tricuspid regurgitation in patients with D-TGA after atrial switch repair and ccTGA [17]. 
Fig. 5 A 42-year-old woman with pulmonary regurgitation after surgically repaired tetralogy of Fallot who presented with dyspnoea at exertion. Early (a) and end (b) diastolic short-axis steady-state free precession cine-MR images showing dilatation of the right ventricle and flattening of the ventricular septum (arrow) at early diastole that regained a normal appearance at the end of diastolic filling. c Phase velocity-encoded cine MR image on a plane perpendicular to the main pulmonary artery during diastole, showing back blood flow, which indicates retrograde flow due to pulmonary regurgitation. The regurgitation rate calculated for this patient was $59 \%$
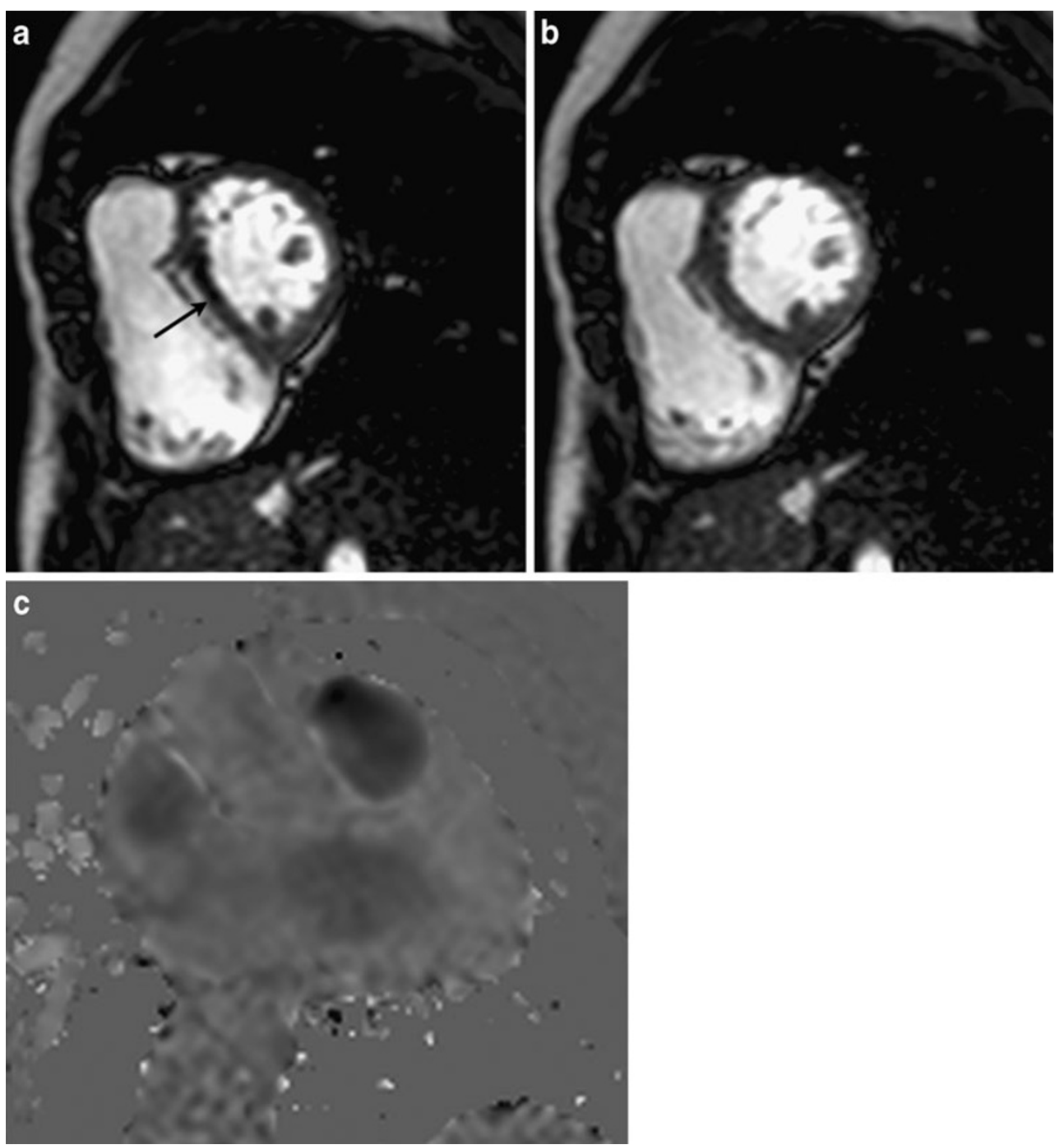

\section{Constriction vs. restriction}

\section{Constrictive pericarditis}

The most distinctive feature of constrictive pericarditis is increased pericardial stiffness, leading to impaired ventricular filling with a subsequent rise in filling pressures. Although a pericardial width of more than $4 \mathrm{~mm}$ has been used as a morphological criterion to diagnose constrictive pericarditis, a significant number of patients with pericardial constriction are found to have a minimally increased or even normal pericardial width during surgery. In such cases, one of the most useful criteria for differentiating constrictive pericarditis from other entities with increased filling pressure, such as restrictive cardiomyopathy, is the presence of abnormal septal bowing towards the LV during early diastole (Movie 3 and Fig. 8) [3]. Early diastolic leftwards ventricular septal bowing can be enhanced at the onset of inspiration as the changes in intrathoracic pressure are not transmitted to the cardiac chambers, because the non-compliant pericardium impedes the outward movement of the ventricular free wall (Movie 3). Therefore, during inspiration, the pulmonary venous left atrial pressure gradient decreases, reducing flow to the left heart, with a concomitant increase in flow to the right chambers, a sign of pathological ventricular interdependence $[3,18]$.

Inspiratory septal bowing during early diastole can also be seen in inflammatory pericarditis (Fig. 9) because the inflammatory thickened pericardial layers may lower pericardial compliance and lead to transient pericardial constriction [18]. Late enhancement of the inflamed pericardium after the administration of gadolinium-based contrast material is useful to differentiate inflammatory pericarditis from fibrosing forms of chronic pericarditis (Fig. 9b).

\section{Abnormal electrical activity}

The conduction delay existing in the left bundle branch block is generally associated with delayed depolarisation and contraction of the lateral LV free wall, causing the 


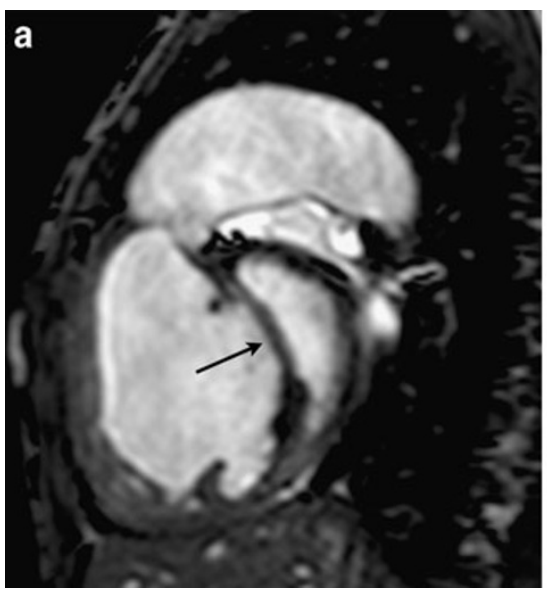

Fig. 6 An 18-year-old man with severe idiopathic pulmonary hypertension presented with haemoptysis. Short-axis steady-state free precession cine MR image at early diastole (a) and end diastole (b) shows a huge pulmonary trunk (42 $\mathrm{mm}$ ), dilated and hypertrophied right ventricle and septal leftwards bowing during early diastole (arrow) that regained a

septum to move passively to the LV cavity in early systole. Furthermore, tricuspid valve opening and right ventricular filling occur much earlier than mitral valve opening and left ventricular filling, and the resultant additional RV volume may be responsible for the early systolic displacement of the septum into the left ventricle. When the rest of the left ventricle contracts, the septum is relaxed and bows into the right ventricle because of rising left ventricular pressure. This paradoxical motion does not cause any mechanical disadvantage in structurally normal hearts [19].

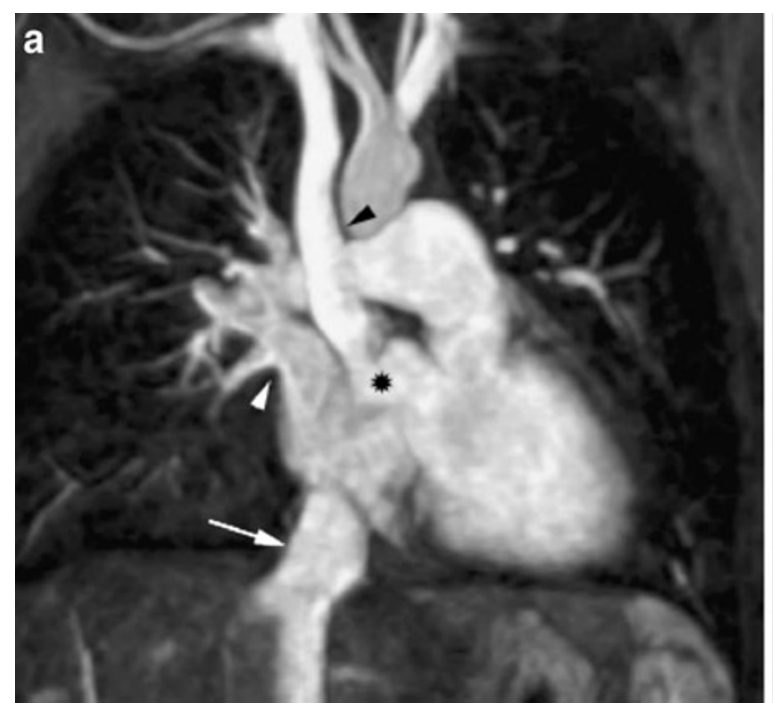

Fig. 7 A 15-year-old girl presenting with increasing dyspnoea; the patient had undergone an atrial switch operation in infancy for dextrotransposition of the great arteries. a Coronal maximum intensity projection image showing the pulmonary vein (white arrowhead) to right atrium communication and drainage of the superior (black arrowhead) and inferior (arrow) vena cava into systemic, surgically

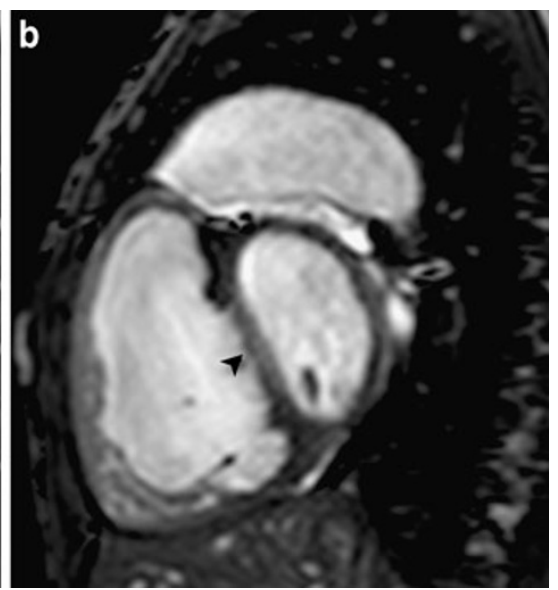

flattened appearance (arrowhead) at the end of diastolic filling. Systolic gradient in the pulmonary trunk obtained from velocity-encoded cine MR images was $65 \mathrm{mmHg}$ and volumetric MR quantification revealed systolic dysfunction of the right ventricle (ejection fraction, 20\%) and left ventricle (ejection fraction, 40\%; not shown)

The presence of left bundle branch block in patients with dilated or ischaemic cardiomyopathy implies a progressive worsening of the LV systolic function and prognosis. The early right ventricular activation leads to right ventricular ejection during the left ventricular enddiastolic period and the septal motion is paradoxical. Consequently there is a reduced LV filling, decreased septal contribution and increased functional mitral regurgitation, which ultimately decreases forward cardiac output [20].

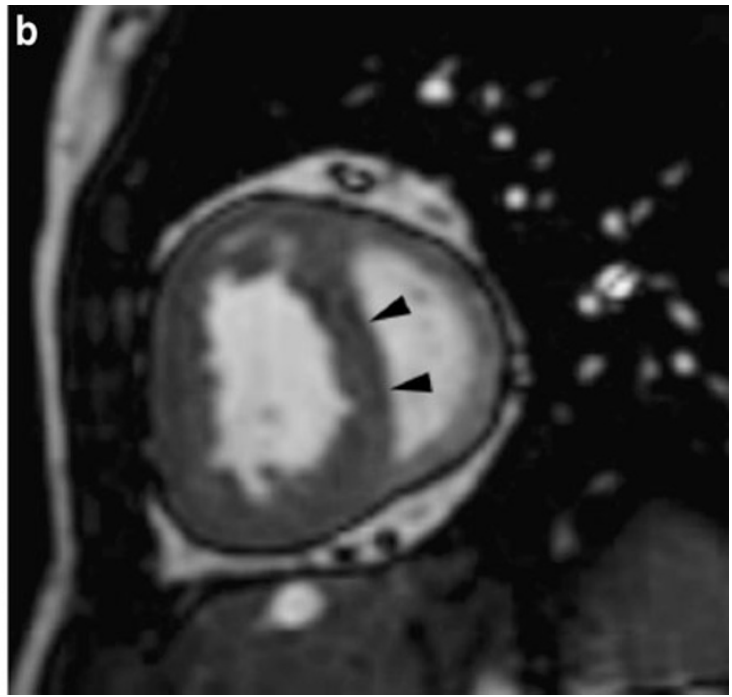

constructed venous atrial baffle (asterisk), isolating the mitral valve from pulmonary venous drainage. b Short-axis cine-MR image obtained at systole showing a circular enlarged systemic right ventricle, a crescentic left ventricle and leftwards bowing of the ventricular septum (arrowheads). Volumetric MR quantification (not shown) yielded a right ventricular ejection fraction of $37 \%$ 


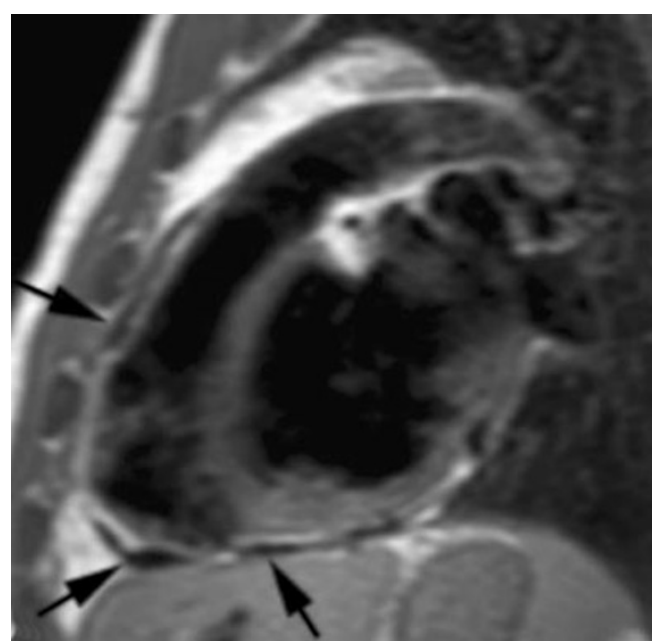

Fig. 8 A 36-year-old man who had had symptoms of right heart failure for 2 months. Sagittal T1-weighted fast spin-echo MR image showing diffuse pericardial thickening (arrows) outlined by epicardial and anterior mediastinal fat, which was most pronounced over the right ventricle

\section{Septal myocardial disease}

Myocardial infarction of the septum

Left anterior descending artery occlusion produces necrosis of the apex and anterior septum.

Septal myocardial infarction has many deleterious effects, among which are the loss of contractile function, impairment of electrical conduction and loss of ventricular synchrony [21].
Left bundle branch block is commonly associated with atherosclerotic coronary artery disease, and its identification in these patients is important to stratify the risk and manage the therapy [22].

Patients with antero-septal myocardial infarction and left bundle branch block exhibit similar paradoxical systolic movement of the IVS (Fig. 10), which may limit the interpretation of non-invasive stress tests [23]. Moreover, myocardial perfusion studies often suffer from falsepositive antero-septal or septal perfusion defects in the absence of left anterior descending artery stenosis [22].

Only myocardial contrast echocardiography with adenosine and 64-slice computed tomography seems to improve diagnostic accuracy [22].

Recent studies have shown that tagging cardiac MRI combined with viability data obtained by late gadolinium enhancement may be a valuable adjunct for the assessment of myocardial viability in patients with regional wall motion abnormalities and left bundle branch block or myocardial infarction (Fig. 10c) [23].

\section{Arrhythmogenic right ventricular cardiomyopathy}

The septum plays a central role in the understanding and management of the RV failure seen in RV dysplasia. The disease involvement is not limited only to the RV as LV has also been reportedly affected. Right ventricular free wall is replaced by a fatty deposition that allows the free wall to dilate and become aneurysmal. These right ventricular

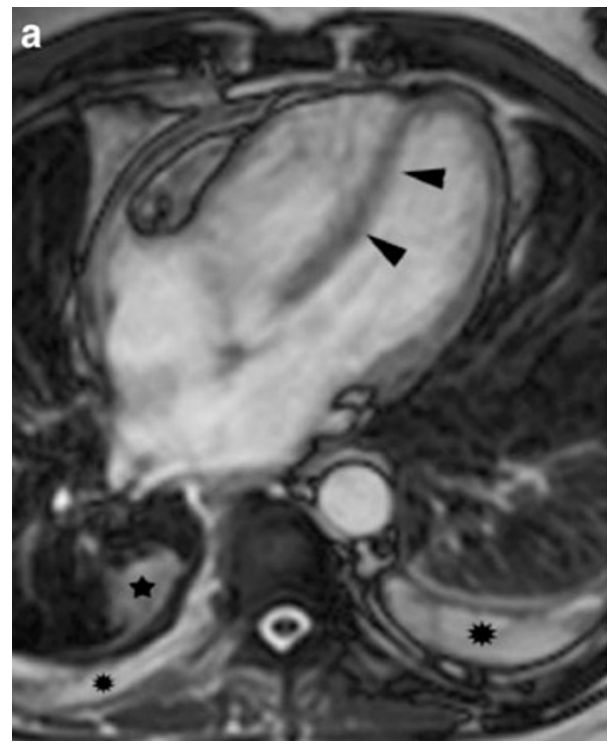

Fig. 9 A 60-year-old man with shortness of breath and chest pain. a Early diastolic four-chamber steady-state free precession cine-MR image during operator-guided breathing inspiration, showing ventricular septal flattening (arrowheads) that disappears at late filling (not shown). Note the associated small pericardial effusion and bilateral pleural effusions (asterisks). A round atelectasis (star) can also be observed. b

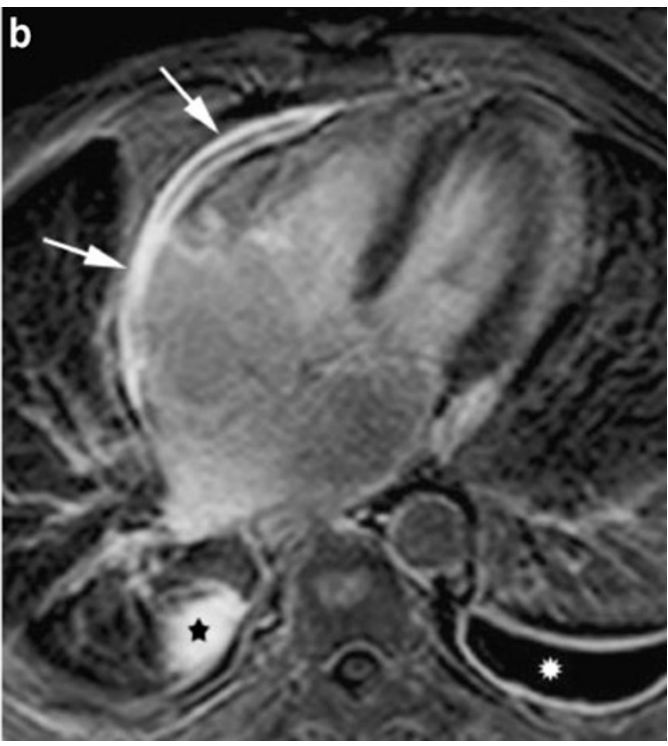

Four-chamber inversion-recovery turbo field-echo MR image revealing delayed enhancement of the pericardium (arrows), indicative of inflammation. Note the associated small pericardial effusion and bilateral pleural effusions (asterisk) and round atelectasis (star). Findings at subsequent pericardiocentesis confirmed exudative effusion 

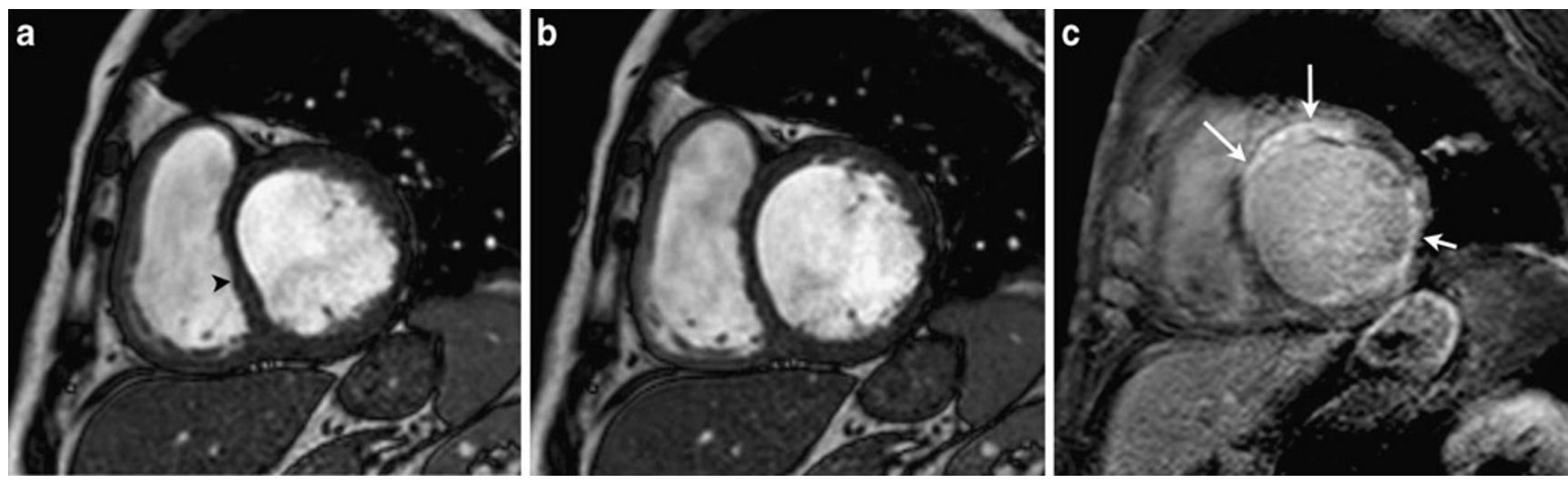

Fig. 10 A 48-year-old man with myocardial infarction and left branch block bundle. Short-axis steady-state free precession cine-MR image obtained at basal level in early systole (a) and end diastole (b) show leftwards septal shift during early systole (arrowhead) and normal

changes exist despite normal pulmonary artery pressure. Left ventricular involvement is associated with increased myocardial mass, inflammatory infiltrates, clinical arrhythmic events and more severe right ventricular wall thinning and heart failure [24].

Cardiac MRI can show normal septal function or septal flattening or leftwards bowing during early diastole, which may lead to left ventricular dysfunction. Understanding septal function and its contribution to RV performance has allowed for a rational design of a procedure to treat this disease. The treatment goals are to limit free wall aneurysm, as reported experimentally, and restore the midline septal position [25].

\section{Multi-factorial causes}

Abnormal septal motion due to multi-factorial causes may also be observed after cardiac surgery and cardiac transplant.

\section{Cardiac surgery}

Systolic movement of the interventricular septum towards the LV despite normal thickening is assumed to be a usual and inevitable event after uncomplicated heart valve surgery, coronary artery bypass grafting and cardiac transplantation. The cause is uncertain but is likely to be related to alterations in heart mobility in the chest due to postoperative adhesions, conduction abnormalities and some mechanism that injures the IVS. In general, PSM is typically transient, not due to septal ischaemia or infarct, and resolves within the first year as pericardial adhesions form or as conduction abnormalities [5].

Leftwards septal bowing during systole can also be seen in cardiac transplant patients (Fig. 11) with RV pressure configuration of the interventricular septum at end diastole. c Shortaxis inversion-recovery turbo field-echo MR image obtained at basal level revealing delayed transmural enhancement (arrows) involving the anterior, antero-septal and infero-lateral wall

overload in the donor heart secondary to the effect of the recipient's increased pulmonary resistance by chronic LV failure of the excised heart [26].

\section{Conclusion}

Cardiac MRI assessment of ventricular septal curvature in systole and diastole is important for evaluating the haemodynamic status in patients with congenital and acquired heart disease in routine clinical practice.

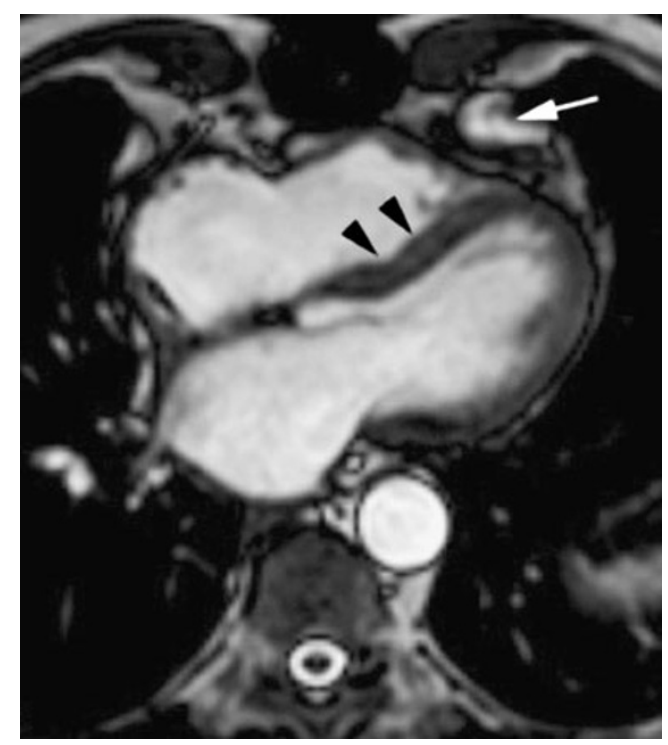

Fig. 11 A 64-year-old man with right ventricular dysfunction 4 months after cardiac transplantation. Four-chamber steady-state free precession cine MR image obtained at early-diastole, showing leftwards bowing of the posterior ventricular septum (arrowheads). A small mediastinal fluid collection (arrow) is also visible 
Paradoxical septal wall motion can be seen in structurally normal hearts with a history of left bundle branch block or is typically transient after open cardiac surgery. However, with the presence of abnormal septal motion, additional emphasis should be placed on the confirmation, as well as determination, of the aetiology and severity of right-sided pressure and/or volume overload. Future studies are required to show the predictability of septal wall motion abnormality and the degree of cardiac dysfunction.

\section{References}

1. Saleh S, Liakopoulos OJ, Buckberg GD (2008) The septal motor of biventricular function. Eur J Cardiothorac Surg 29(Suppl 1): S126-S138

2. Joshi SB, Salah AK, Mendoza DD, Goldstein SA, Fuisz AR, Lindsay J (2009) Mechanism of paradoxical ventricular septal motion after coronary artery bypass grafting. Am J Cardiol 103:212-215

3. Giorgi B, Mollet NR, Dymarkowski S, Rademakers FE, Bogaert J (2003) Clinically suspected constrictive pericarditis: MR imaging assessment of ventricular septal motion and configuration in patients and healthy subjects. Radiology 228:417-424

4. Grosberg A, Gharib M, Kheradvar A (2009) Effect of fiber geometry on pulsatile pumping and energy expenditure. Bull Math Biol 71:1580-1598

5. Reynolds HR, Tunick PA, Grossi EA, Dilmanian H, Colvin SB, Kronzon I (2007) Paradoxical septal motion after cardiac surgery: a review of 3,292 cases. Clin Cardiol 30:621-623

6. Roeleveld RJ, Marcus JT, Faes TJ, Gan TJ, Boonstra A, Postmus PE, Vonk-Noordegraaf A (2005) Interventricular septal configuration at MR imaging and pulmonary arterial pressure in pulmonary hypertension. Radiology 234:710-717

7. Abd El Rahman MY, Hui W, Dsebissowa F, Schubert S, Gutberlet M, Hetzer R, Lange PE, Abdul-Khaliq H (2005) Quantitative analysis of paradoxical interventricular septal motion following corrective surgery of tetralogy of Fallot. Pediatr Cardiol 26:379-384

8. Louie EK, Rich S, Levitsky S, Brundage BH (1992) Doppler echocardiographic demonstration of the differential effects of right ventricular pressure and volume overload on left ventricular geometry and filling. J Am Coll Cardiol 19:84-90

9. Kardesoglu E, Cebeci BS, Celik T, Cingozbay BY, Dincturk M, Demiralp E (2004) Assessment of interventricular septal motion using colour tissue Doppler imaging in adult patients with atrial septal defect. J Int Med Res 32:14-18

10. Davlouros PA, Niwa K, Webb G, Gatzoulis MA (2008) The right ventricle in congenital heart disease. Heart 92(Suppl 1):i27-i38

11. Attenhofer Jost CH, Connolly HM, Dearani JA, Edwards WD, Danielson GK (2007) Ebstein's anomaly. Circulation 115:277285

12. Zoghbi WA, Enriquez-Sarano M, Foster E, Grayburn PA, Kraft CD, Levine RA, Nihoyannopoulos P, Otto CM, Quinones MA, Rakowski H, Stewart WJ, Waggoner A, Weissman NJ (2003) American Society of Echocardiography. Recommendations for evaluation of the severity of native valvular regurgitation with two-dimensional and Doppler echocardiography. J Am Soc Echocardiogr 16:777-802
13. Buckberg GD, RESTORE Group (2006) The ventricular septum: the lion of right ventricular function, and its impact on right ventricular restoration. Eur J Cardiothorac Surg 29:S272-S278

14. Lurz P, Puranik R, Nordmeyer J, Muthurangu V, Hansen MS, Schievano S, Marek J, Bonhoeffer P, Taylor AM (2009) Improvement in left ventricular filling properties after relief of right ventricle to pulmonary artery conduit obstruction: contribution of septal motion and interventricular mechanical delay. Eur Heart J 30:2266-2274

15. Dellegrottaglie S, Sanz J, Poon M, Viles-Gonzalez JF, Sulica R, Goyenechea M, Macaluso F, Fuster V, Rajagopalan S (2007) Pulmonary hypertension: accuracy of detection with left ventricular septal-to-free wall curvature ratio measured at cardiac MR. Radiology 243:63-69

16. Love BA, Mehta D, Fuster VF, Medscape (2008) Evaluation and management of the adult patient with transposition of the great arteries following atrial-level (Senning or Mustard) repair. Nat Clin Pract Cardiovasc 5:454-467

17. Kral Kollars CA, Gelehrter S, Bove EL, Ensing G (2010) Effects of morphologic left ventricular pressure on right ventricular geometry and tricuspid valve regurgitation in patients with congenitally corrected transposition of the great arteries. Am J Cardiol 105:735-739

18. Francone M, Dymarkowski S, Kalantzi M, Rademakers FE, Bogaert $\mathrm{J}$ (2006) Assessment of ventricular coupling with real-time cine MRI and its value to differentiate constrictive pericarditis from restrictive cardiomyopathy. Eur Radiol 16:944-951

19. Grines CL, Bashore TM, Boudoulas H, Olson S, Shafer P, Wooley CF (1989) Functional abnormalities in isolated left bundle branch block. The effect of interventricular asynchrony. Circulation 79:845-853

20. Ansalone G, Giannantoni P, Ricci R, Trambaiolo P, Fedele F, Santini M (2003) Biventricular pacing in heart failure: back to basics in the pathophysiology of left bundle branch block to reduce the number of nonresponders. Am J Cardiol 91(9A):55F$61 \mathrm{~F}, 8$

21. Karavidas AI, Matsakas EP, Lazaros GA, Brestas PS, Avramidis DA, Zacharoulis AA, Fotiadis IN, Korres DA, Zacharoulis AA (2006) Comparison of myocardial contrast echocardiography with SPECT in the evaluation of coronary artery disease in asymptomatic patients with LBBB. Int J Cardiol 112:334-340

22. Ghostine S, Caussin C, Daoud B, Habis M, Perrier E, Pesenti-Rossi D, Sigal-Cinqualbre A, Angel CY, Lancelin B, Capderou A, Paul JF (2006) Non-invasive detection of coronary artery disease in patients with left bundle branch block using 64-slice computed tomography. J Am Coll Cardiol 48:1929-1234, 21

23. Rutz AK, Manka R, Kozerke S, Roas S, Boesiger P (2009) Schwitter J (2009) Left ventricular dyssynchrony in patients with left bundle branch block and patients after myocardial infarction: integration of mechanics and viability by cardiac magnetic resonance. Eur Heart J 30:2117-2127

24. Jain A, Tandri H, Calkins H, Bluemke DA (2008) Role of cardiovascular magnetic resonance imaging in arrhythmogenic right ventricular dysplasia. J Cardiovasc Magn Reson 20:10-32

25. Frigiola A, Giamberti A, Chessa M, Di Donato M, Abella R, Foresti S, Carlucci C, Negura D, Carminati M, Buckberg G, Menicanti L (2006) RESTORE group. Right ventricular restoration during pulmonary valve implantation in adults with congenital heart disease. Eur J Cardiothorac Surg 29:S279-S285

26. Nwakanma LU, Shah AS, Conte JV, Baumgartner WA (2008) In: Cohn LH (ed) Heart transplantation in cardiac surgery in the adult. McGraw-Hill, New York, pp 1539-1578 